XIV Российская национальная конференция по сейсмостойкому строительству и сейсмическому районированию (с международным участием). Сборник материалов

УДК 624.94.012.45:624.044.3:699.841

\title{
DOI 10.37153/2687-0045-2021-14-101-106
}

\section{УТОЧНЕНИЕ КОЭФФИЦИЕНТА К 1 ДЛЯ РАСЧЕТОВ ЖЕЛЕЗОБЕТОННЫХ РАМНЫХ КАРКАСОВ НА ВОЗДЕЙСТВИЕ ТИПА РЗ (ПО РЕЗУЛЬТАТАМ НАТУРНОГО ВИБРАЦИОННОГО ЭКСПЕРИМЕНТА)}

\author{
Соснин A.B., \\ Научно-исследовательская лаборатория \\ оценки безопасности результатов проектирования и \\ сейсмостойкости строительных конструкций, \\ (214000, г. Смоленск, ул. Ленина, д.13А) \\ Центральный научно-исследовательский и проектный институт \\ жилых и общественных зданий (ЦНИИЭП жилища), \\ (129090, г. Москва, просп. Мира, д. 16, стр. 2) (syabryauskas@mail.ru)
}

Аннотация: С применением диаграммы деформирования железобетонного каркаса (без вертикальных диафрагм и связей жесткости) рассмотрены особенности количественной оценки коэффициента допускаемых повреждений $\mathrm{K}_{1}$. Частный объект исследования фрагмент жилой блок-секции из 4 этажей размерами в осях 14,7×9,6 м, возведенный в г. Ашхабаде, испытан в 1968 году сотрудниками ЦНИИЭП жилища под руководством Шапиро Г.А. с применением вибрационной машины В-2. Над объектом исследования автором проведен численный эксперимент с применением концепции метода нелинейного статического (Pushover) анализа в SAP2000; проведены расчетные оценки сейсмостойкости с применением критериев наступления предельного состояния, близкого к обрушению. Проведена оценка зависимости параметров ниспадающей ветви диаграммы деформирования каркаса от параметров армирования зон пластичности в его элементах. Полученные результаты сопоставлены с результатами зарубежных натурных испытаний на фрагментах железобетонных каркасов. Расчетами установлено, что допускаемому уровню повреждений каркаса соответствует коэффициент $\mathrm{K}_{1}$, равный 0,34, при относительном горизонтальном перекосе нижнего этажа 1,85 \%. Выявлено, что значение для $\mathrm{K}_{1}$, указанное в СП 14.13330 для объектов исследования соответствует области больших перемещений диаграммы деформирования системы, поэтому не может соответствовать цели расчета подобных каркасов на воздействие типа РЗ. Установлено, что резервы сейсмостойкости в объектах исследования обусловливаются в основном параметрами поперечного армирования зон пластичности. Отмечается необходимость дополнения СП 14.13330.2018 требованиями к минимальному значению коэффициента поперечного армирования в зонах пластичности несущих элементов, примыкающих к узлам рам железобетонных каркасов. Поясняется, что дефицит сейсмостойкости $\mathrm{I}_{\mathrm{d}}$ в объектах исследования, подобных рассматриваемому, формируется из-за отсутствия согласованности $\mathrm{K}_{1}$ с расчетными параметрами и специальными требованиями по конструированию зон пластичности.

Ключевые слова: железобетонный рамный каркас, здания с «гибким» этажом, концепция нелинейного статического анализа, диаграмма деформирования здания, параметры армирования зон пластичности, критерии сейсмостойкости по необрушению, коэффициент допускаемых повреждений $\mathrm{K}_{1}$, натурный вибрационный эксперимент 
XIV Российская национальная конференция по сейсмостойкому строительству и сейсмическому районированию (с международным участием). Сборник материалов

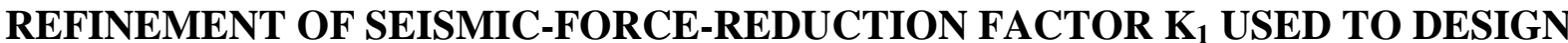 OF RC MOMENT FRAMES LOADED BY SEISMIC FORCES CONFORMING OF A DESIGN EARTHQUAKE (BASED ON AN IN-SITU FULL-SCALE TEST)
}

\author{
Sosnin A.V. \\ Scientific-Research Laboratory \\ of Design Outcomes Safety Estimation and \\ Earthquake-Resistance of Building Structures \\ (Ofc. No. 27, Bldg 13A, Lenina St, Smolensk, 214000, Russian Federation) \\ Central Research Institute for Experimental Design \\ (CNIIEHP), Moscow, Russian Federation \\ (Bldg 16/2, Pr-t Mira, Moscow, 129090, Russian Federation)
}

\begin{abstract}
Quantitative estimation features of seismic-force-reduction factor $\mathrm{K}_{1}$ for a reinforced concrete moment frame (without shear walls and braces) using a Pushover curve are considered. The research object is a 4-story unit of a residential building with sizes on axis $14,7 \times 9,6 \mathrm{~m}$, erected in Ashgabat; tested in 1968 by representatives of Central Research Institute for Experimental Design (the Russian acronym, - CNIIEHP) under leadership of G.A. Shapiro with a powerful vibrating machine type B-2. A computational experiment under the frame had been recently conducted by the author; estimations were performed using the non-linear static Pushover analysis methodology in SAP2000 software. For the frame earthquake-resistance assessments using criteria of limit state closed to collapse were performed. An estimation of parameters dependence of a recessive part of a Pushover curve of the frame on reinforcement parameters of hinges zones in its RC members is carried out. Results of the work were compared with tests results obtained foreign researchers accomplished on RC frames fragments. Calculation estimates have established that an allowed limit state for the frame corresponds to seismic-force-reduction factor $\mathrm{K}_{1}$ is equal to 0.34 , at a relative story drift of ground floor with value 1.85 percent. It was revealed that the value for $\mathrm{K}_{1}$ indicated in Building Seismic Design Code SP14.13330 for study objects corresponds to a large displacements area of the frame Pushover curve, therefore, $\mathrm{K}_{1}$ cannot correspond to a purpose of a calculating procedure using Response Spectrum Method for similar frames which perceptive seismic loads formed by a design earthquake (named with the Russian acronym,-RZ). It was found that having of earthquake-resistance reserves in the study objects is mainly due to transverse/web reinforcement parameters in hinge zones. It is noted, that it is necessary to supplement of SP 14.13330 with requirements for a minimum value of web reinforcement in hinges zones of primary members adjacent to beam-column joints of RC moment frames. It is explained that an earthquakeresistance shortage in similar study objects is formed due to absence of conformity of K1-factor with computational parameters and special design requirements of hinge zones.
\end{abstract}

Keywords: RC frame, soft-story buildings, Pushover-analysis conception, Pushover curve, hinge zones reinforcement parameters, ultimate earthquake-resistance criteria, seismic-force-reduction factor $\mathrm{K}_{1}$ (in Seismic Building Design Code SP 14.13330.2014 formulation), in-situ vibrating test

Цель исследования - продолжить разработку метода идентификации нелинейных механических систем по результатам натурных вибрационных экспериментов с применением способов расчетного построения диаграмм деформирования реальных объектов, основываясь на концепции нелинейного статического анализа; ${ }^{(i)}$ продолжить исследования по развитию методологии уточнения значений коэффициента допускаемых повреждений $\mathrm{K}_{1}$ для обеспечения его согласованности с механизмом пластического 
XIV Российская национальная конференция по сейсмостойкому строительству и сейсмическому районированию (с международным участием). Сборник материалов

деформирования, образующимся в объектах исследования при сильном землетрясении, и дифференциации значений $\mathrm{K}_{1}$ в зависимости от условий строительства и параметров рассматриваемой системы.

Любое здание или сооружение может подвергнуться воздействию землетрясения, которое для него будет сильным событием с позиции реализации допускаемых повреждений. Возможные соотношения между коэффициентом $\mathrm{K}_{1}$ и коэффициентом редукции $\mathrm{R}$ рассмотрены автором в работе [1]. При оценке сейсмостойкости системы с применением кривой равновесных состояний формата «сейсмическая сдвигающая сила в уровне основания - перемещение перекрытия верхнего этажа» $\left(\mathrm{V}_{\text {sh.b. }}-\Delta\right)$, характерной реакции сооружения каркасного типа, выявляются следующие особенности. Инженерный анализ последствий прошлых землетрясений показал, что в железобетонных каркасных зданиях повреждения, обуславливающие уровень LS, соответствуют области больших перемещений. Полная потеря эксплуатационных характеристик каркаса соответствует положению точки упруго-пластических свойств в начале пологого участка кривой равновесных состояний вблизи правой границы уровня повреждений с индексом $\mathrm{OI}_{(\mathrm{R})}$. Тогда получается, что для учета в расчетах повреждений, характерных уровню $\mathrm{OI}_{(\mathrm{R})}$, инженеру предписано для коэффициента $\mathrm{K}_{1}$ принимать значение, фактически соответствующее уровню повреждений LS (пояснено в работе [1]). Для подтверждения указанных пояснений с применением концепции метода нелинейного статического (Pushover) анализа проведен численный эксперимент над железобетонным каркасом из 4 этажей (объект № 10 в таблице 10 [2]). По критериям, представленным в работе [3], рассматриваемый объект исследования полностью соответствует области применения Pushover-методологии. В основу сопоставительного анализа результатов численного и натурного эксперимента заложено сравнение параметров диаграммы деформирования каркаса, полученной при проведении натурного эксперимента, с кривыми равновесных состояний, полученных автором в SAP2000. Результаты численного эксперимента позволили уточнить теоретические оценки $\mathrm{R}_{\mu}$, $\mathrm{R}, \mathrm{K}_{1}$, ранее опубликованные автором в работах $[1,4]$.

При проведении натурного эксперимента ниспадающая ветвь диаграммы деформирования для каркаса была получена только теоретически с применением выражения (1):

$$
Q=\alpha A_{\text {макс }}^{x}-\beta A_{\text {макс }}^{2}
$$

Отмечается, что на ниспадающей ветви диаграммы деформирования, полученной по выражению (1), отсутствует пологий участок, обычно соответствующий упругопластической работе узлов сопряжения колонн с ригелями. Автором определено, что диаграмма деформирования каркаса, построенная после проведения натурного эксперимента (включая ниспадающую ветвь), практически идентично аппроксимируется кривой, удовлетворяющей зависимости (2):

$$
f(x)=a\left(1-e^{-\frac{x}{b}}-c x\right),
$$

где a, b и с-численные коэффициенты, вычисленные равными $92,0.83$ и 0.16 соответственно.

Способность каркаса к редуцированию сейсмических сил оценивалась посредством численного моделирования нелинейной работы расчетных участков зон пластичности несущих элементов в месте примыкания к узлам каркаса. Влияние зон пластичности учитывалось путем применения диаграмм состояния формата «момент - кривизна» М- 
XIV Российская национальная конференция по сейсмостойкому строительству и сейсмическому районированию (с международным участием). Сборник материалов

$\varphi$ (фи). Для учета свойств сжатого бетона использовалась диаграмма деформирования Mander'a (рассмотрена в [5]). Учитывалось, что основной вклад в формирование параметров траектории поиска положения точки упруго-пластических свойств вносит демпфирование $\xi_{0}$, соответствующее гистерезисному затуханию. Кривая равновесных состояний и оценки сейсмической реакции получены методом спектра несущей способности S. Freeman'a. Суммарный угол искажения рамного узла определяется углом перелома оси элементов каркаса, примыкающих к узлу. При получении требуемого значения коэффициента поперечной арматуры $\rho_{s t}$ (англ. volumetric transverse/web reinforcement ratio) принято выражение (3):

$$
\rho_{s t}=\max \left(0.3 \frac{f_{c}^{\prime}}{f_{y t}}\left(\frac{A_{g}}{A_{c}}-1\right) ; 0.09 \frac{f_{c}^{\prime}}{f_{y t}}\right),
$$

В качестве критерия обеспечения сейсмостойкости (критерия оценки результатов экспериментов) учитывалась не максимальная сдвигающая сила в уровне основания,

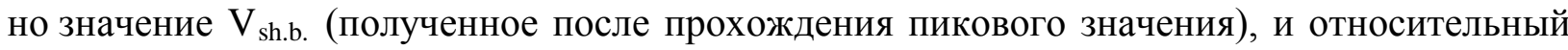
горизонтальный перекос этажа. Для уровня IO значение относительного перекоса принималось в интервале $0,18 \%-0,79 \%$; для уровня повреждений LS - 1,15\%-2,79\%. Наступление предельного состояния, характерного уровню повреждений LS, оценивалось по критерию Duan'a L. и Cooper'a T.R. (1995).

Для подтверждения объективности результатов проведенного численного эксперимента рассмотрены результаты натурных испытаний зарубежных исследователей на фрагментах железобетонных каркасов (без диафрагм и связей жесткости), доведенных до предельного состояния, которые провели Ockleston A.J. (1956), Lee H.S., Woo S.W., Heo Y.S., Song J.G. (1999), Pinho R. и Elnashai A.S. (2000), Calvi G.M., Magenes G. и Pampanin S. (2002), $\quad$ Di Sarno L. и Manfredi G. (2009), $\quad$ Dolce M., $\quad$ Cardone D., Ponzo F.C. и Valente C. (2005), Jeong S.-H. и Elnashai A.S. (2004), и др.

Гипотеза, результаты численного эксперимента, основные результаты исследования, основные критерии соответствия и главные выводы представлены автором на международной конференции «Комплексный подход к расчетам строительных конструкций и оснований» (НИП-Информатика, Москва, 15.11.2019 г.), на международной научно-практической конференции «Проектирование и расчеты 2021» (НИП-Информатика, Москва, 22-26.03.2021 г.), на научно-практическом семинаре «Научно-техническая экспертиза и сопровождение строительных проектов: проблемы, достижения, перспективы» (ЦНИИП Минстроя России, Москва, 13.04.2021г.), и подробно изложены в работе [6].

Анализ полученных результатов позволил сделать вывод, что расчетная упругопластическая модель каркаса адекватно описывает реальный физический эксперимент. Результаты численного и натурного экспериментов хорошо дополняют друг друга. Восходящая ветвь и начало пологого участка диаграммы деформирования каркаса (кривой равновесных состояний), полученной в SAP2000, соответствуют траектории нелинейной диаграммы деформирования, построенной при испытании каркаса вибрационной машиной В-2. Численный эксперимент позволил выявить влияние параметров поперечной арматуры на вид диаграммы деформирования каркаса в области больших перемещений, в то время как в результатах натурного эксперимента эта важная особенность не была получена. Сравнивая результаты экспериментальных и теоретических исследований, можно отметить хорошую сходимость до уровня $\mathrm{IO}_{(\mathrm{R})}$; Заметное расхождение кривых начинается, как только прогиб каркаса превышает значение 55 мм. Наклон пологого участка кривых равновесных состояний к оси абсцисс обуславливается реализацией в каркасе «гибкого» нижнего этажа и влиянием эффектов второго рода. 
XIV Российская национальная конференция по сейсмостойкому строительству и сейсмическому районированию (с международным участием). Сборник материалов

В случае доведения каркаса до предельного состояния реализуется механизм пластического деформирования, определяемый как non-ductile-column-sway mechanism, по сценарию схожий с реакцией зданий с так называемым «гибким» нижним этажом (что согласуется с пояснениями, представленными на с. 132-134 [2], и зарубежным опытом расчета сейсмостойких конструкций). При допущении уровня повреждений IO, соответствующего потере эксплуатационных свойств, значение коэффициента, интегрально учитывающего повреждения несущих конструкций при сейсмическом воздействии, определено равным 0,54. Если допускать уровень повреждений LS, обеспечивающий безопасность жизнедеятельности, то для $\mathrm{K}_{1}$ определяется значение 0,34 . Последнее из указанных значений с некоторыми допущениями удовлетворяет правилам проектирования в сейсмических районах, но при этом реакция испытываемого каркаса не соответствует требованиям к первоочередному образованию зон пластичности в несущих элементах железобетонных каркасов. Реакция каркаса характеризуется коэффициентом редукции $\mathrm{R}$ равным 2,95, что характерно системам со слабым армированием несущих конструкций, относящимся к категории ordinary moment-resisting frame. При рассмотрении требований СП 14.13330.2014, можно сделать вывод, что нормативное значение коэффициента $\mathrm{K}_{1}$, регламентируемое для объекта исследования, не обеспечивает соблюдение цели расчета на сейсмическое воздействие РЗ. Для упразднения указанного несоответствия предлагается, для расчетов железобетонных рамных каркасов на воздействие типа Р3 коэффициент $\mathrm{K}_{1}$ назначать равным не менее 0,5.

При проведении численного эксперимента установлено, что поперечные хомуты в зонах пластичности каркаса начинают вносить вклад в процесс редуцирования сейсмических сил только после реализации в нижнем этаже перекоса со значениями, превышающими $0,6 \%$. В случае ограничения перекоса нижнего этажа только значением 1/350 поперечная арматура и длина критических участков зон пластичности в несущих элементах не успевают оказать свое влияние на ниспадающую ветвь диаграммы деформирования системы. Способность такого каркаса к редуцированию сейсмических сил при проектировании с применением линейно-спектрального метода по СП 14.13330.2018 практически не получается оценить расчетом. Плато на диаграмме деформирования исследуемого каркаса проявилось только при шаге хомутов 150мм. При наложении кривой спектра реакции (характерного уровню воздействия типа КЗ) на кривую равновесных состояний четко определяется влияние поперечной арматуры на положение точки свойств системы, что свидетельствует о применимости подхода, предложенного автором в работе [5].

Для обеспечения коэффициента $\mathrm{K}_{1}$ с целью проектирования железобетонных каркасных зданий, требования к устройству (подбору параметров, расположению и конструированию) зон пластичности, содержащиеся в разделе 6.8 СП 14.13330.2018, следует расширить, детализировать и согласовать с положениями расчетного раздела указанного свода правил. Для реализации требований абзаца 3 пункта 6.8.1 СП 14.1330.2014 необходимо ввести требования к минимальному коэффициенту поперечного армирования железобетонных элементов, примыкающих к узлам рам железобетонных каркасов. Для этого предлагается применять выражение (3).

В железобетонных зданиях и сооружениях, обладающих низкой пластичностью, величина $\mathrm{K}_{1}$ может оказывать влияние на формирование дефицита сейсмостойкости $\mathrm{I}_{\mathrm{d}}$ уже на стадии проектирования. Опираясь на результаты проведенного исследования, можно сделать вывод, что дефицит сейсмостойкости $\mathrm{I}_{\mathrm{d}}$ в объектах исследования, подобных рассматриваемому, формируется из-за отсутствия согласованности $\mathrm{K}_{1}$ с расчетными параметрами и специальными требованиями по конструированию зон пластичности несущих элементов. При этом дефицит сейсмостойкости будет зависеть не от уровня положения плато расчетного спектра реакции, а от того, насколько высоко 
XIV Российская национальная конференция по сейсмостойкому строительству и сейсмическому районированию (с международным участием). Сборник материалов

будет приподнята «хвостовая» часть спектра (при построении спектра реакции формата $\mathrm{Sa}_{\mathrm{T}}$ в линейном масштабе) [7].

\section{Список литературы}

1. Соснин А.В. Об уточнении коэффициента допускаемых повреждений $\mathrm{K}_{1}$ и его согласованности с концепцией редукции сейсмических сил в постановке спектрального метода (в порядке обсуждения) // Вестник гражданских инженеров. 2017. №1(60). С. 92114.

2. Шапиро Г.А., Симон Ю.А., Ашкинадзе Г.Н., Захаров В.Ф., Барков Ю.В.

Вибрационные испытания зданий / Госкомитет по делам строительства и архитектуры при Госстрое СССР, ЦНИИЭП жилища; под ред. Г.А. Шапиро. М.: Стройиздат, 1972. 160 с.

3. Соснин А.В. Об особенностях методологии нелинейного статического анализа и его согласованности с базовой нормативной методикой расчета зданий и сооружений на действие сейсмических сил // Вестник ЮУрГУ. Серия «Строительство и архитектура». 2016. Т. 16. № 1. С. 12-19.

4. Соснин A.В. К вопросу учета диссипативных свойств многоэтажных железобетонных каркасных зданий массового строительства при оценке их сейсмостойкости // Современная наука и инновации. 2017. № 1(17). С. 127-144.

5. Соснин А.В. Методика двухстадийного расчета армирования элементов железобетонных каркасных зданий и сооружений на действие сейсмических сил с применением концепции нелинейного статического анализа. Часть 1: Постановка задачи, структура методики, информационная база исследования и стратегия определения параметров зон пластичности // Вестник ЮУрГУ. Серия «Строительство и архитектура». 2018. T. 18. № 1. C. 5-31.

6. Соснин А.В. Сопоставление допускаемых повреждений железобетонных каркасных зданий с целью расчетов на сейсмические воздействия // Жилищное строительство. 2021. № 1-2. С. 50-80.

7. Соснин А.В. Детерминированная оценка дефицита сейсмостойкости железобетонных каркасов // Вестник ЮУрГУ. Серия «Строительство и архитектура». 2021. Т. 21, № 2. С. 12-31. 